\title{
Análise da associação entre oclusão e disfunção temporomandibular: estudo clínico
}

\author{
Analysis of the association between occlusion and temporomandibular \\ dysfunction: clinical study \\ Análisis de la asociación entre oclusión y disfunción temporomandibular: \\ estudio clínico \\ Sandro Basso BITENCOURT ${ }^{\mathbf{1}}$ \\ Ana Issa de Oliveira CUNHA ${ }^{2}$ \\ Emily Vivianne Freitas da Silva² \\ Aline Terra Biazon JARDIM²
}

${ }^{1}$ Faculdade de Odontologia de Araçatuba, Univ Estadual Paulista, UNESP 16015.050 Araçatuba-SP, Brasil

${ }^{2}$ Curso de Odontologia, Universidade Anhanguera UNIDERP 79003.010 Campo Grande-MS, Brasil

\begin{abstract}
Resumo
Com o objetivo de verificar a associação entre a oclusão e a disfunção temporomandibular (DTM), o presente estudo se propôs a avaliar a oclusão com relação às Seis Chaves de Oclusão de Andrews, os guias de desoclusão e os contatos dentais, em pacientes com a presença associada ou não de DTM, seguindo o Research Diagnostic Criteria (RDC). Foram incluídos 18 pacientes, de ambos os sexos, com faixa etária entre 21 e 61 anos, os quais participaram da fase clínica (análise do RDC) e da fase laboratorial (análise da oclusão). Na análise de todos os pacientes, os considerados com boa oclusão e com DTM foram $17 \%$ e sem DTM 6\%, com distúrbios oclusais e com DTM foram 33\% e sem DTM 44\%. Com base na metodologia aplicada, nos resultados obtidos nesta análise pode-se concluir que não houve relação entre pacientes que apresentaram distúrbios oclusais e obrigatoriamente DTM.
\end{abstract}

Descritores: Oclusão Dentária; Transtornos da Articulação Temporomandibular; Má Oclusão.

\begin{abstract}
In order to verify the association between occlusion and temporomandibular dysfunction (TMD), the present study aimed to evaluate the occlusion with respect to the Six Andrews Keys of Occlusion, the disocclusion guides and the dental contacts, in patients with TMD or not, following the Research Diagnostic Criteria (RDC). We were included 18 patients of both genders, aged between 21 and 61 years, whom participated in the clinical phase (RDC analysis) and the laboratory phase (occlusion analysis). In the analysis of all patients, those considered with good occlusion and TMD were 17\% and 6\% without TMD, those with occlusal disorders and TMD were $33 \%$ and 44\% without TMD. Based on the methodology; on the results of this analysis and on the most recent articles in the literature, we can conclude that there wasn't relation between patients who had occlusal disorders and necessarily TDM.

Descriptors: Dental Occlusion; Temporomandibular Joint Disorders; Malloclusion.

\section{Resumen}

Con el objetivo de verificar la asociación entre oclusión y disfunción temporomandibular (TTM), el presente estudio tuvo como objetivo evaluar la oclusión con respecto a las seis llaves de oclusión de Andrews, las guías de disociación y los contactos dentales, en pacientes con TTM o no, siguiendo el Criterios Diagnósticos de Investigación (RDC). Se incluyeron 18 pacientes de ambos sexos, con edades comprendidas entre 21 y 61 años, que participaron en la fase clínica (análisis RDC) y la fase de laboratorio (análisis de oclusión). En el análisis de todos los pacientes, los que se consideraron con buena oclusión y TMD fueron $17 \%$ y $6 \%$ sin TMD, aquellos con trastornos oclusales y TMD fueron $33 \%$ y 44\% sin TMD. Basado en la metodología; sobre los resultados de este análisis y sobre los artículos más recientes en la literatura, podemos concluir que no hubo relación entre pacientes que tenían desórdenes oclusales y necesariamente TDM.
\end{abstract}

Descriptores: Oclusión Dental; Trastornos de la Articulación Temporomandibular; Maloclusión.

\section{INTRODUÇÃO}

A oclusão normal é descrita como uma relação harmoniosa entre dentes da maxila e da mandíbula, onde temos uma oclusão estável, sã e esteticamente atrativa ${ }^{1-3}$. A maloclusão, por sua vez, constitui uma alteração do desenvolvimento dentário e/ou dos arcos dentários, ocasionando problemas estéticos e/ou funcionais, tendo como causas mais comuns as condições funcionais adquiridas ${ }^{4}$.

O termo disfunção temporomandibular (DTM) tem sido comumente utilizado para descrever a associação de determinados aspectos clínicos que geram distúrbios ao sistema mastigatório ${ }^{5,6}$. A literatura aponta que a DTM representa uma série de problemas clínicos envolvendo a articulação temporomandibular (ATM) e/ou a musculatura mastigatória e estruturas relacionadas. Apesar de ser tradicionalmente vista como uma síndrome, atualmente é considerada como um conjunto de desordens relacionadas ao sistema mastigatório. A característica mais comum é a dor localizada nos músculos mastigatórios ou na região da ATM, geralmente agravada durante a mastigação ou movimentos mandibulares. Outros sintomas incluem dores de ouvido, cefaleia e dores na face. Quanto aos sinais clínicos, apresentam ruídos na articulação e limitações durante os movimentos mandibulares ${ }^{5,7-12}$.
A idade pode ser considerada como um fator de risco para DTM considerando-se algumas particularidades. Em pacientes idosos, pode-se observar um aumento da prevalência de sinais clínicos e radiográficos, em contrapartida, observa-se também uma menor prevalência de sintomas e de demanda de tratamento comparado ao verificado em adultos jovens. O sexo também parece ser um fator de risco, uma vez que a DTM também é mais frequente em mulheres. Ainda como fatores de risco, têm-se os hábitos parafuncionais e o bruxismo, bem como os aspectos psicológicos. Os fatores genéticos e o tratamento ortodôntico não têm sido evidenciados como causa de DTM ${ }^{7,13,14}$.

Até o presente momento, não foi estabelecida uma clara relação entre alterações oclusais e DTM, tendo sido apenas observado que a desarmonia entre a relação cêntrica e a máxima intercuspidação, a mordida cruzada unilateral e a ausência de estabilidade oclusal causada pela perda de dentes posteriores, parecem ter evidenciado certo potencial preditivo ${ }^{15}$. Há muita controvérsia em relação ao papel da oclusão como fator etiológico das DTM, pois não há uma definição se a oclusão é o maior fator etiológico das DTM ou se não há íntima relação entre os dois fatores .

Com o objetivo de verificar a associação entre a 
oclusão e a DTM, o presente estudo se propôs a avaliar a oclusão de pacientes dentados e de pacientes parcial ou totalmente edêntulos, com relação à presença ou não das seis chaves de oclusão de Andrews, os guias de desoclusão e os contatos dentais. Em conjunto, foi usado o Research Diagnostic Criteria - Eixo I para o diagnóstico de DTM.

\section{MATERIAL E MÉTODO}

O projeto de pesquisa foi aprovado pelo Comitê de Ética em Pesquisa envolvendo seres humanos da Universidade Anhanguera/Uniderp, sob o protocolo $\mathrm{n}^{\mathrm{o}}$ 012/2012. Pacientes que procuraram atendimento odontológico no Complexo Policlínico Odontológico da Anhanguera/Uniderp, estado de Mato Grosso do Sul, no período de agosto a outubro de 2012, foram escolhidos de forma aleatória, sem distinção de raça e sexo, sendo todos maiores de idade e aptos a responder os questionamentos do RDC. Após a explicação do objetivo da pesquisa e dos passos a serem realizados, foi assinado o Termo de Consentimento Livre e Esclarecido. Foram excluídos da pesquisa aqueles indivíduos que tinham sofrido acidentes e/ou intervenções cirúrgicas na face nos seis meses antecedentes ao estudo; que possuíam desordens mentais ou físicas, que impossibilitavam resposta confiável ao questionário; e que possuíam doenças autoimunes ou desordens degenerativas ou história prévia de otite crônica ou cirurgia otológica.

A pesquisa foi idealizada em duas fases: a fase clínica e a fase laboratorial. A fase clínica constituiu-se em duas etapas: 1) obtenção dos modelos de estudos e montagem do arco facial; 2) análise da presença ou não de DTM e o estabelecimento do diagnóstico do subtipo de DTM, onde foi utilizado o questionário recomendado pela Academia Europeia das Desordens Craniomandibulares e o Research Diagnostic Criteria (RDC) - Eixo $\mathrm{I}^{16}$. O questionário foi considerado uma ferramenta de exclusão dos pacientes sobre a análise de DTM, pois o paciente só passaria à análise pelo RDC quando respondesse afirmativo ao uma pergunta do questionário. Para realização do exame clínico para DTM, o paciente foi posicionado sentado na cadeira odontológica e o examinador à frente do paciente com visualização direta. A fase laboratorial fundamentou-se na montagem dos modelos em Articulador Semi-Ajustável do tipo Arcon (articuladorcôndilo), para posterior avaliação da oclusão através de uma ficha idealizada pelos autores, baseada nos critérios estabelecidos por Angle ${ }^{17}$ e Andrews ${ }^{18}$.

Os materiais utilizados no RDC foram: régua milimetrada de $15 \mathrm{~cm}$ (CIS, Brasil), lapiseira $\mathrm{n}^{\circ}$ 0,5 (Faber Castell, Brasil) e compasso de ponta seca (Jon, Brasil). Para obtenção dos modelos de estudo foram utilizados: jogo de moldeiras para pacientes dentados (Tecnodent, Brasil), cera utilidade (Wilson, Brasil) para individualização da moldeira, alginato Jeltrate Dustless $®$ (Dentsply, Brasil), espátula e cuba de borracha (Indusbello, Brasil) para espatular o material de moldagem, cera $\mathrm{n}^{\circ} 7$ (Wilson, Brasil) para o registro de mordida, lamparina a álcool (JON), gesso especial Durone ${ }^{\circledR}$ (Dentsply, Brasil) e gesso comum tipo II (Polident, Brasil). Para montagem em articulador, foi usado um Articulador Semi-Ajustável do tipo Arcon (DentFlex, Brasil), godiva (DFL, Brasil) para registro da mordida no arco facial e gesso comum para fixação dos modelos no articulador.

$\mathrm{Na}$ fase laboratorial, depois de concluída a montagem dos modelos no articulador, foi usada a ficha anteriormente citada, analisando a presença ou não das seis chaves de oclusão de Andrews, as guias de desoclusão e o tipo de contatos dentais, através de pinça Müller (SSWhite, Brasil) e papel carbono Accufilm II (Parkell, Brasil). Dois examinadores foram calibrados a fim de padronizar o exame clínico e as mensurações, auxiliando na confiabilidade da pesquisa. A estatística do estudo foi do tipo descritiva, sendo os dados analisados por intermédio do programa estatístico
EpiInfo versão 5.2, cujo qual trabalha com dados em um campo de $95 \%$ de confiabilidade, ou seja, fornece margem de $5 \%$ de erro.

Com relação à classificação entre pacientes com boa oclusão ou com distúrbios oclusais, consideramos com boa oclusão os pacientes que apresentaram cinco ou seis chaves de oclusão de Andrews, contatos bilaterais simultâneos e que não tinham interferências oclusais. Com distúrbios oclusais foram classificados os pacientes que estiveram fora destes critérios de boa oclusão.

\section{RESULTADOS}

Um total de 18 pacientes foi incluído no estudo, com idade média 28,11 anos e mediana de 22,5 anos, significando que dentre os pacientes pesquisados havia mais pacientes jovens. Quanto ao sexo, obtivemos um total de 15 pacientes do sexo feminino $(83,3 \%)$ e 3 do sexo masculino $(16,7 \%)$, demonstrando uma predominância de mulheres participando da pesquisa (Tabela 1).

Tabela 1. Distribuição do sexo dos pacientes incluídos
\begin{tabular}{|c|c|c|}
\hline Sexo & $\mathbf{n}$ & $\%$ \\
\hline Feminino & 15 & $83,3 \%$ \\
\hline Masculino & 3 & $16,7 \%$ \\
\hline Total & 18 & $100 \%$ \\
\hline
\end{tabular}

Ao analisarmos pacientes parcial ou totalmente edêntulos, os resultados mostram que os pacientes edêntulos $(\mathrm{n} .=2)$ apresentaram resultados distintos com relação à DTM, onde 50\% (n=1) apresentaram DTM e 50\% (n. 1) não apresentaram sintomas de DTM, mesmo todos apresentando características de distúrbios oclusais, como ausência de dentes posteriores e próteses removíveis mal adaptadas (Tabela 2).

Tabela 2. Dados demográficos dos pacientes edêntulos incluídos no estudo

\begin{tabular}{|c|c|c|c|c|c|c|}
\hline Edêntulo & $\%$ & $\mathbf{n}$ & Sexo & Idade & Oclusão & Diagnóstico \\
\hline Com DTM & 50 & 1 & Feminino & 52 & $\begin{array}{c}\text { Distúrbios } \\
\text { oclusais }\end{array}$ & $\begin{array}{c}\text { DM, DDCR, } \\
\text { Artralgia }\end{array}$ \\
\hline Sem DTM & 50 & 1 & Feminino & 61 & $\begin{array}{c}\text { Distúrbios } \\
\text { oclusais }\end{array}$ & Sem sintomas \\
\hline
\end{tabular}

Quando considerados os pacientes dentados, importante destacar que as classes de Angle e as chaves de oclusão de Andrews foram avaliadas apenas nos pacientes dentados $(n=16)$, excluindo pacientes parcial ou totalmente edêntulos (n.=2). A Classe I mostrou-se presente em 56,3\% $(\mathrm{n} .=9)$ dos pacientes. A Classe II esteve em 43,8\% (n=7) dos analisados. Nenhum paciente, nem do sexo masculino quanto do feminino, apresentou mordida Classe III. Com base nestes dados, nossa amostra teve predominância de pacientes com mordida classe I.

A análise da presença das seis chaves de oclusão de Andrews (Figura 1) foi um dos critérios de classificação de uma boa oclusão. Dentre os 16 pacientes dentados, 25\% $(n=4)$ foram considerados pacientes com uma boa oclusão, por conter no mínimo cinco chaves.

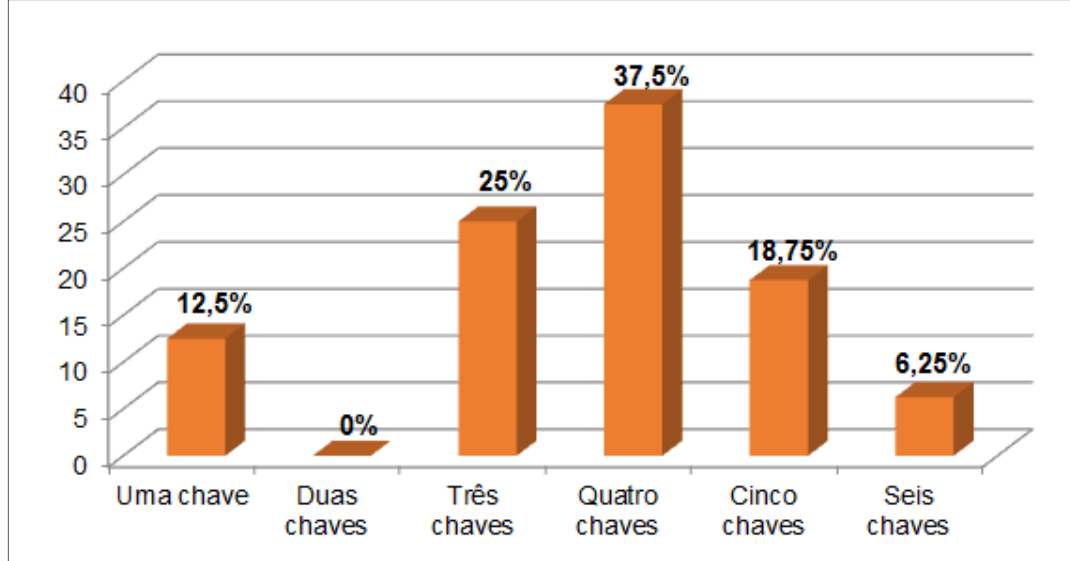

Figura 1: Distribuição das Chaves de Oclusão entre os pacientes incluídos. 
Os pacientes que apresentaram quatro chaves ou menos foram classificados com distúrbios oclusais, totalizando $75 \%($ n.=12) (Figura 2).



Figura 2: Dados demográficos da distribuição dos pacientes classificados com boa oclusão e com distúrbios oclusais

Com relação ao diagnóstico de DTM (Figura 3), o diagnóstico mais prevalente foi Dor Miofascial, em 30,8\% dos pacientes $(\mathrm{n}=8)$, sendo todos do sexo feminino. $\mathrm{O}$ diagnóstico de Deslocamento de Disco com Redução esteve presente em $19,2 \%$ dos pacientes $(n=5)$, também sendo todos do sexo feminino. A Artralgia esteve em $11,5 \%$ dos pacientes (n.=3), todos do sexo feminino. Já o Deslocamento de Disco sem Redução sem Limitação de Abertura significou 3,8\% dos pacientes $(\mathrm{n}=1)$, todos do sexo feminino. Pacientes sem classificação foram 3,8\% (n=1), sendo apenas do sexo masculino. Pacientes sem sintomas de DTM totalizaram 30,8\% (n=8). Diagnósticos de Dor Miofascial com Limitação de Abertura, Deslocamento de Disco sem Redução com Limitação Abertura, Osteoartrite e Osteoartrose da ATM não foram computados, pois nenhum dos pacientes pesquisados apresentou sinais e sintomas destes diagnósticos.

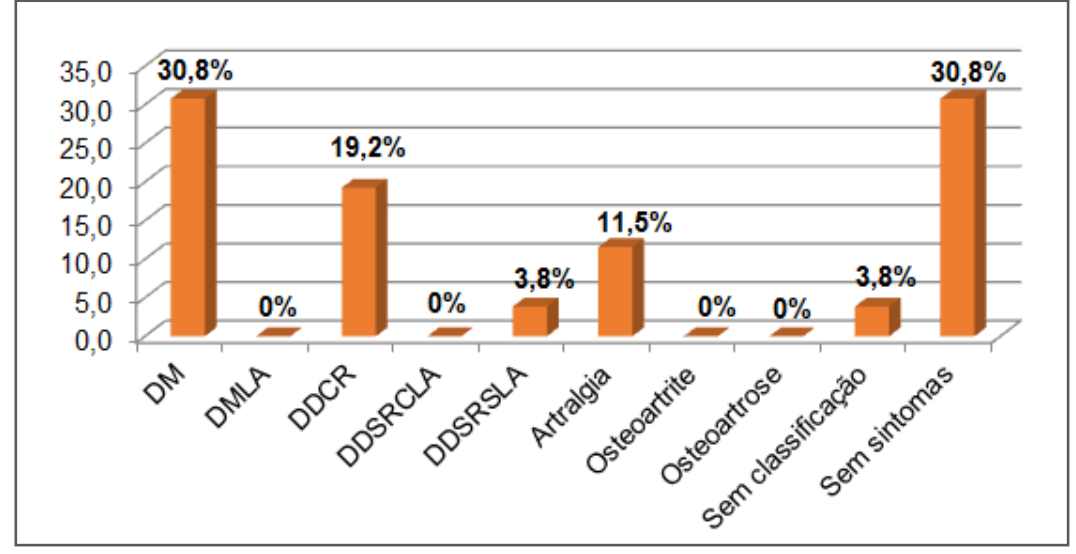

Figura 3: Diagnósticos DTM.

Com relação ao diagnóstico de DTM comparado ao sexo, apenas pacientes do sexo feminino apresentaram sintomas de DTM classificáveis $(65,4 \%)$. Um paciente do sexo masculino apresentou sintomas de DTM (4,3\%), porém com base nos dados obtidos, não foi possível a classificação em um diagnóstico específico (Figura 4). A média de idade dos pacientes de ambos os sexos que apresentaram DTM foi de 28,44 anos.

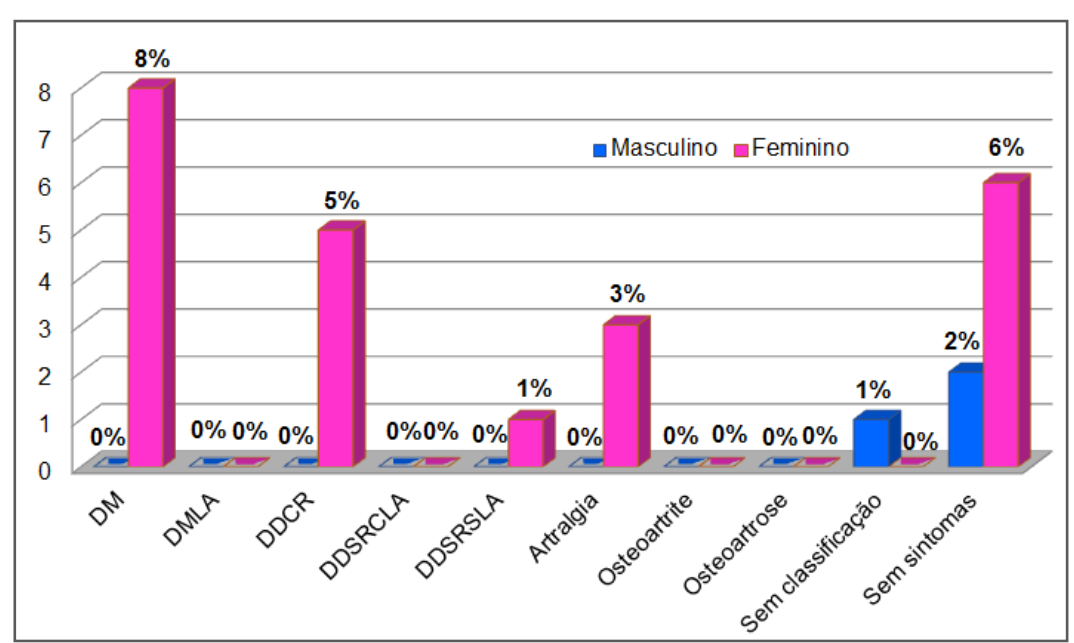

Figura 4: Diagnósticos de DTM X Sexo.
$\mathrm{Na}$ análise de todos os pacientes, os considerados com boa oclusão e com DTM foram 17\% (n. 3) e sem DTM 6\% (n.1), com distúrbios oclusais e com DTM foram $33 \%$ (n. 6) e sem DTM 44\% (n. 8) (Figura 5).

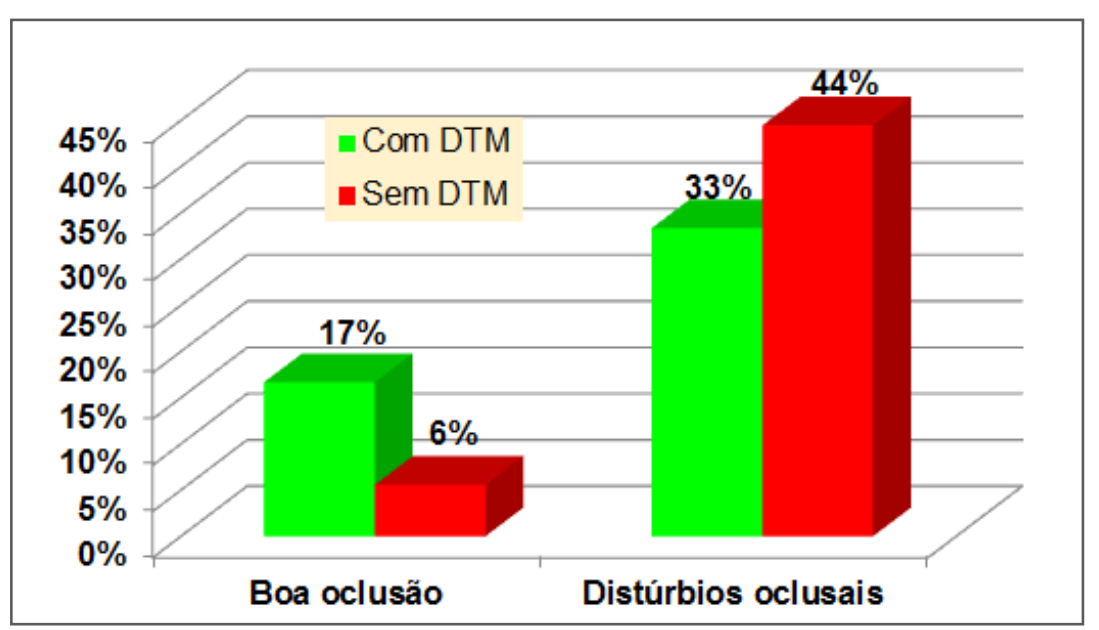

Figura 5: Distribuição da análise de DTM em pacientes considerados com boa oclusão ou com distúrbios oclusais.

\section{DISCUSSÃO}

A correlação entre distúrbios oclusais e DTM ainda é amplamente discutida na literatura, sendo motivo de controvérsias e necessitando de um consenso a fim de padronizar um plano de tratamento tanto para as oclusopatias, quanto para as DTM. A oclusão normal, satisfatória, dinâmica e estética para Andrews $^{18}$, deve seguir as seis chaves de oclusão por ele preconizadas para alcance de tal diagnóstico oclusal. Para Pacífico ${ }^{19}$, a definição de uma oclusão boa ou normal deveria ser considerada como normal ideal, sendo que a apresentação de dentes intercuspidados e posicionados dentro de uma linha de oclusão impecável é de baixa frequência. Assim, o autor acrescenta que seria inadequado o uso do termo "normal", pois o mesmo demonstra apenas uma condição adaptativa que admite variações, enquanto que o ideal representa uma condição hipotética para alcance. Cohen ${ }^{20}$ descreve a predileção pelo termo oclusão satisfatória, ao invés de normal ou ideal, além de escolher desarmonias oclusais à maloclusão, pois o último carrega certo significado patológico que nem sempre acontece. Brangeli ${ }^{21}$ demonstra que, apesar de haver referência comprovada no uso das chaves de Andrews para análise oclusal, grande parte das pesquisas opta pelo método de classificação de Angle, tal fato que explica a baixa prevalência das seis chaves em oclusões denominadas como normais nos estudos observados.

Em nosso estudo, para realizar o diagnóstico de DTM, o protocolo RDC/TMD - eixo I foi empregado, pois permite a análise precisa de variáveis clínicas, como dor à palpação, ruídos articulares e padrão de abertura bucal, por ser um mecanismo universalmente aceito e válido para vários idiomas, além da confiabilidade comprovada dos métodos de avaliação clínica definidos ${ }^{22,23}$.

O ambiente intrabucal não é o ideal para avaliar os padrões de contato dentário, as interferências e as várias relações de deslizamento da mandíbula, bem como, o sistema neuromuscular do paciente é frequentemente programado para evitar determinados padrões de contato que se tenta discernir $^{24}$. Diante disso, o mecanismo utilizado para auxiliar no exame detalhado da condição oclusal foi o Articulador Semi-Ajustável do tipo Arcon, o qual é um instrumento mecânico que representa a ATM, a maxila e a mandíbula ${ }^{25}$. Por meio de modelos de estudo adequadamente montados em articulador, torna-se possível executar os movimentos bordejantes da mandíbula sem a influência do sistema neuromuscular, auxiliando na visualização do relacionamento estático e dinâmico dos dentes, sendo possível analisar regiões de difícil acesso na boca, como os posteriores e porção lingual da oclusão, permitindo rever as relações das cúspides e fossas de suporte ${ }^{25,26}$. Contudo, existem erros inerentes na reprodução, exigindo precisão e controle de 
todos os aspectos na produção dos modelos a serem montados no articulador ${ }^{24}$, pois vários passos são tomados até a montagem completa dos modelos, que vai desde a obtenção dos modelos de estudo à montagem do arco facial e a instalação final de todos os componentes no articulador, demandando atenção durante todo o processo, a fim de evitar erros que possam alterar o resultado final no momento da análise oclusal.

A média etária de pacientes com DTM neste estudo foi de 28,33 anos, o que é comprovada pela pesquisa de Teixeira et al. ${ }^{27}$, que encontraram uma maior frequência de pacientes com DTM na faixa etária de 20 a 29 anos e por Okeson ${ }^{9}$ que afirmou que a faixa etária de maior prevalência para DTM é de 20 a 40 anos. O sexo prevalente na pesquisa com DTM, foi o feminino, totalizando $87 \%$ dos diagnósticos estabelecidos pelo RDC, ratificado pelo estudo de Gesch et al. ${ }^{28}$ e Marinho et al. $^{23}$, onde afirmaram que a DTM é mais prevalente em mulheres. Com relação ao diagnóstico mais prevalente, a Dor Miofascial esteve presente em 30,8\% dos pacientes, sendo todos do sexo feminino, onde Graff-Radford et al. ${ }^{29}$ comentam que a dor miofascial é um dos diagnósticos mais comuns na classificação da DTM.

Barbosa et al. ${ }^{15}$, em sua revisão de literatura, observaram que as disfunções temporomandibulares intraarticulares possuem alguma associação com os tipos de maloclusões classificadas por Angle, independente do seu tipo. Isto foi comprovado em nossa pesquisa, onde todos os pacientes classificados como classe II $(37,5 \%)$ foram diagnosticados com algum tipo de DTM, enquanto que nos pacientes classe I apenas 18,8\% foram diagnosticados e $43,8 \%$ não apresentaram sinais ou sintomas indicativos de DTM, indicando que há prevalência maior de DTM em pacientes com maloclusão classe II.

Muitos indivíduos adaptam-se às alterações oclusais dentro dos limites toleráveis, sem o aparecimento de sinais e sintomas mastigatórios, e essas alterações podem ser de importância secundária e originar sintomas apenas após o avanço da DTM, a qual seria originada por outros fatores ${ }^{15}$. Pullinger e Seligman ${ }^{30}$, ao analisarem 381 pacientes em modelos de gesso, concluíram que a oclusão dentária é responsável por apenas uma pequena parcela da amostra. Para Bósio $^{31}$, o resultado dos últimos estudos indica que se deve tomar muito cuidado ao dizer que problemas oclusais são a causa dos problemas de DTM. Corroboramos com essas afirmações, pois durante as análises, encontramos uma paciente com perda de vários elementos posteriores superiores e inferiores, com os dentes anteriores em abertura em leque, sem uso de próteses removíveis e sem nenhuma queixa de dor muscular e/ou articular. Sugerindo ainda, a não relação direta entre oclusão e DTM, nosso estudo obteve resultados significativos quando comparada a oclusão com DTM, onde $17 \%$ dos pacientes considerados com boa oclusão foram diagnosticados com DTM e apenas $6 \%$ não apresentaram sintomas de DTM. Quando verificamos os pacientes com distúrbios oclusais, 33\% tinham DTM e $44 \%$ ficaram com o grupo dos sem DTM. Com isso, pode-se sugerir que a oclusão pode não ter relação direta na etiologia de DTM, podendo ser mais uma consequência do que uma causa, assim como sugerido anteriormente por Pullinger e Seligman $^{30}$.

Dentro do campo de pesquisa, uma das limitações do estudo foi não haver um protocolo estandardizado de execução para análise da oclusão, diferente do diagnóstico de DTM, que por sua vez, apresentou confiabilidade esclarecida em um segmento idealizado e comprovado. Assim, a associação entre as diferentes observações, tornou-se incompleta para oclusão. Porém, depois de um estudo amplo, considerando as principais características a estudar, uma ficha de análise para direcionar o espectro da pesquisa foi confeccionada. A resultante idealizada foi uma ficha composta pelas seis chaves de Andrews, classificação de Angle, tipo de mordida, guias de desoclusão e contatos dentais. Em pacientes parcial ou totalmente edêntulos os critérios já se apresentaram como tipo de prótese utilizada, guias de desoclusão e contatos dentais.

\section{CONCLUSÃO}

Com base na metodologia aplicada e nos resultados desta análise, pode-se concluir que não houve relação entre pacientes que apresentaram distúrbios oclusais e obrigatoriamente DTM. Vale ressaltar que a literatura ainda é contraditória quando se fala em oclusão e DTM, pois uns afirmam que a DTM não tem nenhuma relação com a oclusão, outros alegam que a maloclusão é uma das principais causas de DTM e já os mais conscientes dizem que a oclusão e DTM andam em conjunto, onde se um sistema for alterado, o outro responde, ou seja, sugerem que há uma relação de causa e efeito mútua entre duas partes de um sistema maior e mais complexo, que é o sistema estomatognático.

\section{REFERÊNCIAS}

1. Angle EH. Classification of malocclusion. Dental Cosmos. 1899; 41(18):248-64.

2. Ferreira FV. Ortodontia. 3.ed., São Paulo: Artes Médicas; 1999.

3. Bumann A, Lotzman U. Disfunção Temporomandibular: Diagnóstico Funcional e Princípios Terapêuticos. Porto Alegre: Artmed, 2002.

4. Tomita NE, Bijela VT, Franco LJ. Relação entre os hábitos bucais e má-oclusão em pré-escolares. Rev Saúde Pública. 2000; 34(3):299-303.

5. Auvenshine RC. Temporomandibular disorders: Associated features. Dent Clin North Am. 2007; 51(1):105-27.

6. Fricton J. Myogenous temporomandibular disorders: diagnostic and management considerations. Dent Clin North Am. 2007; 51(1):61-83.

7. Agerberg G, Carlsson GE. Functional disorders of the masticatory system. I. Distribution of symptoms according to age and sex as judged from investigation by questionnaire. Acta Odontol Scand. 1972; 30(6):597-613.

8. Magnusson T, Egermark I, Carlsson GE. A longitudinal epidemiologic study of signs and symptoms of temporomandibular disorders from 15 to 35 years of age. J Orofac Pain. 2000; 4(14):310-19.

9. Okeson JP. Tratamento das desordens temporomandibulares e oclusão. 4.ed., São Paulo: Artes Médicas; 2000.

10. Rantala MA, Ahlberg J, Suvinen TI, Savolainen A, Konomen M. Symptoms, signs, and clinical diagnoses according to the research diagnostic criteria for temporomandibular disorders among Finnish multiprofessional media personnel .J Orofac Pain. 2003; 17(4):311-16.

11. Palla S. Mioartropatias do sistema mastigatório e Dores Orofaciais. São Paulo: Artes Médicas; 2004.

12. Toledo BAS, Capote TSO, Campos JADB. Association between temporomandibular dysfunction and depression. Cienc Odontol Bras. 2008; 11(4):75-9.

13. Egermark I, Carlsson GE, Magnusson T. A 20-year longitudinal study of subjective symptoms of temporomandibular disorders from childhood to adulthood. Acta Odontol Scand. 2001; 59(1):40-8.

14. Poveda Roda R, Bagán JV, Diaz Fernández M, Hernández Bazán SH, Jiménez Soriano YJ. Review of temporoamndibular joint pathology. Part I: Classification, epidemiology and risk factors. Med Oral Patol Oral Cir Bucal. 2007; 12(4):E292-98.

15. Barbosa GAS, Badaró Filho CR, Fonseca RB, Soares CJ, Neves FD, Fernandes Neto AJ. Distúrbios oclusais: 
associação com a etiologia ou uma conseqüência das disfunções temporomandibulares? JBA. 2003; 3(10): 158-63.

16. Dworkin SF, LeResche L. Research diagnostic criteria for temporomandibular disorders: review, criteria, examinations and specifications, critique. J Craniomandib Disord. 1992; 6(4):301-55.

17. Angle EH. Treatment of malocclusion of the teeth: Angles's system. 7th.ed., Philadelphia: S.S. White; 1907.

18. Andrews LF. The six keys to normal occlusion. Am J Orthod. 1972; 62(3):296-309.

19. Pacífico RA. Avaliação das Seis Chaves de Oclusão de Andrews em estudantes brasileiros do município de Marília - SP [dissertação]. Marília: Faculdade de Odontologia da Universidade de Marília (UNIMAR); 2004.

20. Cohen MM. Ortodontia pediátrica e preventiva. Rio de Janeiro: Interamericana; 1977.

21. Brangeli LAM. Prevalência das seis chaves de oclusão de Andrews em jovens brasileiros com oclusão normal. Ver Assoc Paul Cir Dent. 2001; 55(6):411-5.

22. Pereira Júnior FJ, Favilla EE, Dworkin S, Huggins K. Critérios de diagnóstico para pesquisa das disfunções temporomandibulares (RDC/TMD). Tradução oficial para a língua portuguesa. J bras clin odontol integr. 2004. 8(47):384-95.

23. Marinho CC, Cruz FLG, Leite FPP. Correlação entre a oclusão e a disfunção temporomandibular. Revista Odonto. 2009; 17(34):49-55.

24. McNeill, C. Ciência e Prática da Oclusão. 2.ed., São Paulo: Quintessence; 2000.

25. Maciel RN. Oclusão e ATM - Procedimentos clínicos. São Paulo: Livraria Santos; 1998.

26. Okeson JP. Fundamentos de Oclusão e Desordens Temporo-mandibulares. 2.ed., São Paulo: Artes Médicas; 1992.

27. Gesch D, Bernhardt O, Kocher T, Mack F, Hensel E, Alte D. Association of malocclusion and functional occlusion with signs of temporomandibular disorders in adults: results of the population-based Study of Health in Pomerania. Angle Orthod. 2004; 74(4):512-20.

28. Teixeira ACB, Marcucci G, Luz JGC. Prevalência das maloclusões e dos índices anamnésicos e clínicos, em pacientes com disfunção da articulação temporomandibular. Rev Odontol Univ São Paulo. 1999; 13(3):251-56.

29. Graff-Radford SB, Reeves JL, Jaeger B. Management of chronic head and neck pain: effectiveness of altering factors perpetuating myofascial pain. Headache. 1987; 27(4):186-90.

30. Pullinger AG, Seligman DA. Quantification and validation of predictive values of occlusal variables in temporomandibular disorders using a multifactorial analysis. J Prosthet Dent. 2000; 83(1):66-75.

31. Bósio JA. O paradigma da relação entre oclusão, ortodontia e disfunção têmporo-mandibular. R Dental Press Ortodon Ortop Facial. 2004; 9(6):84-9.

\section{CONFLITO DE INTERESSES}

Os autores declaram não haver conflitos de interesse.

\section{AUTOR PARA CORRESPONDÊNCIA}

\section{Sandro Basso Bitencourt}

sandrodonto@gmail.com

Submetido em 27/04/2018

Aceito em 30/05/2018 